\title{
About Positivity of Green's Functions for Nonlocal Boundary Value Problems with Impulsive Delay Equations
}

\author{
Alexander Domoshnitsky and Irina Volinsky \\ Department of Mathematics and Computer Science, Ariel University, Ariel, Israel \\ Correspondence should be addressed to Alexander Domoshnitsky; adom@ariel.ac.il
}

Received 18 August 2013; Accepted 22 October 2013; Published 13 February 2014

Academic Editors: K. Ammari, F. J. Garcia-Pacheco, and K. Zhu

Copyright (C) 2014 A. Domoshnitsky and I. Volinsky. This is an open access article distributed under the Creative Commons Attribution License, which permits unrestricted use, distribution, and reproduction in any medium, provided the original work is properly cited.

The impulsive delay differential equation is considered $(L x)(t)=x^{\prime}(t)+\sum_{i=1}^{m} p_{i}(t) x\left(t-\tau_{i}(t)\right)=f(t), t \in[a, b], x\left(t_{j}\right)=\beta_{j} x\left(t_{j}-\right.$ $0), j=1, \ldots, k, a=t_{0}<t_{1}<t_{2}<\cdots<t_{k}<t_{k+1}=b, x(\zeta)=0, \zeta \notin[a, b]$, with nonlocal boundary condition $l x=\int_{a}^{b} \varphi(s) x^{\prime}(s) d s+$ $\theta x(a)=c, \varphi \in L_{\infty}[a, b] ; \theta, c \in R$. Various results on existence and uniqueness of solutions and on positivity/negativity of the Green's functions for this equation are obtained.

\section{Introduction}

Mathematical models with impulsive differential equations attract the topic attention of many researchers (see [1-7]); many important results on boundary value problems and stability of these equations have been obtained. One of possible approaches to study impulsive equations is the theory of generalized differential equations allowing researchers to consider systems with continuous solutions as well as systems with discontinuous solutions and discrete systems in the frame of the same theory [8-15]. In this paper we use the approach proposed in the monograph [1] and developed in [16-20].

Various comparison theorems for solutions of the Cauchy and periodic problems for ordinary differential equations with impulses have been obtained in [17, 21-24]. On the basis of the comparison theorems, tests of stability are proposed in $[25,26]$. Theory of impulsive differential equations and inclusions was presented in the book [27].

Equations with nonlocal boundary conditions are applied in modelling different processes (see, e.g., the recent work of Skubachevskii [28]). Nonlocal problems for nonimpulsive functional differential equations were considered in ([25], Chapter 15). Existence results for nonlocal boundary value problems with impulsive equations are presented in [29-35].
There are almost no results on sign constancy of Green's function for impulsive boundary value problems. Concerning nonlocal impulsive boundary value problems, we know, there are no results about positivity/negativity of Green's function. In this paper we propose results of this sort.

In this paper we consider the following impulsive equation:

$$
\begin{array}{r}
(L x)(t)=x^{\prime}(t)+\sum_{i=1}^{m} p_{i}(t) x\left(t-\tau_{i}(t)\right)=f(t), \\
t \in[a, b], \\
x\left(t_{j}\right)=\beta_{j} x\left(t_{j}-0\right), \quad j=1, \ldots, k, \\
a=t_{0}<t_{1}<t_{2}<\cdots<t_{k}<t_{k+1}=b, \\
x(\zeta)=0, \quad \zeta \notin[a, b],
\end{array}
$$

with different types of boundary conditions:

$$
\begin{aligned}
l x=\int_{a}^{b} \varphi(s) x^{\prime}(s) d s+\theta x(a)=c, & \varphi \in L_{\infty}[a, b] ; \\
& \theta, c \in R, \theta \neq 0,
\end{aligned}
$$




$$
\begin{gathered}
x(a)=c, \\
x(a)=\gamma x(b), \quad \gamma \in R, \gamma \neq 1 .
\end{gathered}
$$

We develop the ideas presented in [17] and we have obtained various results on the existence and uniqueness of solutions for impulsive boundary value problems. The main contribution of the presented paper is the formulation and proof of positivity/negativity conditions for Green's functions for the following impulsive functional differential boundary value problems: (1), (2), (4), and (5); (1), (2), (4), and (6); (1), (2), (4), (7).

\section{Solution's Representation Formulas}

Define the space $D\left(t_{1}, \ldots, t_{k}\right)$ of piecewise continuous functions $x:[a, b] \rightarrow R$, isomorphic to the topological product $L \times R$, where $L$ is the space of measurable essential bounded functions $z:[a, b] \rightarrow R$, by the following equality:

$$
\begin{gathered}
x(t)=\int_{a}^{t} \Omega(t, s) z(s) d s+\omega(t) \alpha, \\
\omega(t)=\sum_{i=1}^{k+1} \chi_{\left[t_{i-1}, t_{i}\right)}(t) \prod_{j=1}^{i} \beta_{i-j}, \\
\Omega(t, s)=\sum_{i=1}^{k+1} \chi_{\left[t_{i-1}, t_{i}\right)}(t) \chi_{\left[t_{i-1}, t_{i}\right)}(s) \beta_{0} \\
+\sum_{i=2}^{k+1} \sum_{r=1}^{i-1} \chi_{\left[t_{i-1}, t_{i}\right)}(t) \chi_{\left[t_{i-1}, t_{i}\right)}(s) \prod_{j=1}^{i-r} \beta_{i-j},
\end{gathered}
$$

where $a=t_{0}<t_{1}<t_{2}<\cdots<t_{k}<t_{k+1}=b$.

It is clear that $x(t)$ is absolutely continuous in $\left(t_{i-1}, t_{i}\right), i=$ $1, \ldots, k+1$, satisfying the equality $x\left(t_{i}\right)=\beta_{i} x\left(t_{i}-0\right)$. It is clear that $x(t)$ has discontinuity of the first kind and is continuous from the right at points $t_{i}, i=1, \ldots, k$.

By [17], the general solution of (1)-(4) has the following representation:

$$
x(t)=C(t, a) x(a)+\int_{a}^{t} C(t, s) f(s) d s .
$$

Theorem 1. If the boundary value problem (1)-(5) is uniquely solvable in the space $D\left(t_{1}, \ldots, t_{k}\right)$ for every essential bounded $f$ and $c \in R$, then its solution can be represented in the form

$$
\begin{aligned}
& x(t) \\
& =\int_{a}^{b} G(t, s) f(s) d s+C(t, a) \frac{c}{\theta+\int_{a}^{b} \varphi(s) C_{s}^{\prime}(s, a) d s},
\end{aligned}
$$

where Green's function $G(t, s)$ of this problem is

$$
\begin{aligned}
& G(t, s) \\
& =C(t, s)-C(t, a) \frac{\int_{s}^{b} \varphi(w) C_{w}^{\prime}(w, s) d w+\varphi(s)}{\theta+\int_{a}^{b} \varphi(w) C_{w}^{\prime}(w, a) d w}
\end{aligned}
$$

Proof. From the boundary condition (5), we obtain

$$
\begin{aligned}
\int_{a}^{b} \varphi(s) x^{\prime}(s) d s+\theta x(a)=c \\
\Longrightarrow x(a)=\frac{c}{\theta}-\frac{1}{\theta} \int_{a}^{b} \varphi(s) x^{\prime}(s) d s .
\end{aligned}
$$

Substituting (12) into (9), we obtain

$$
\begin{aligned}
x(t)= & \frac{C(t, a)}{\theta}\left(c-\int_{a}^{b} \varphi(s) x^{\prime}(s) d s\right) \\
& +\int_{a}^{t} C(t, s) f(s) d s .
\end{aligned}
$$

The Cauchy function $C(t, s)$ can be represented in the following form:

$$
\begin{gathered}
C(t, s)=\sum_{i=0}^{k} \sum_{j=0}^{k} C_{i j}(t, s)\left[H_{t_{i}}(t)-H_{t_{i+1}}(t)\right] \\
\times\left[H_{t_{j}}(s)-H_{t_{j+1}}(s)\right],
\end{gathered}
$$

where $C_{i j}(t, s)=C(t, s)$ in the rectangle $t_{i} \leq t<t_{i+1}, s_{i} \leq s<$ $s_{i+1}$.

Let us take derivative in (9):

$$
\begin{aligned}
x^{\prime}(t)= & C_{t}^{\prime}(t, a) x(a)+\frac{d}{d t}\left(\int_{a}^{t} C(t, s) f(s) d s\right) \\
= & C_{t}^{\prime}(t, a) x(a)+\int_{a}^{t} C_{t}^{\prime}(t, s) f(s) d s \\
& +C(t, t) f(t) .
\end{aligned}
$$

Since, according to the definition of the Cauchy function, $C(t, t)=1$, we obtain

$$
x^{\prime}(t)=C_{t}^{\prime}(t, a) x(a)+\int_{a}^{t} C_{t}^{\prime}(t, s) f(s) d s+f(t) .
$$

Let us substitute (16) into (13) as follows:

$$
\begin{aligned}
& x(t) \\
& =\frac{C(t, a)}{\theta} \\
& \quad \times\left(c-\int_{a}^{b} \varphi(w)\left[C_{w}^{\prime}(w, a) x(a)\right.\right. \\
& \left.\left.\quad+\int_{a}^{w} C_{w}^{\prime}(w, \tau) f(\tau) d \tau+f(w)\right] d w\right) \\
& +\int_{a}^{t} C(t, s) f(s) d s
\end{aligned}
$$




$$
\begin{aligned}
& =\frac{c}{\theta} C(t, a)-\frac{C(t, a)}{\theta} \\
& \times \int_{a}^{b} \varphi(w)\left[C_{w}^{\prime}(w, a) x(a)\right. \\
& \left.+\int_{a}^{w} C_{w}^{\prime}(w, \tau) f(\tau) d \tau+f(w)\right] d w \\
& +\int_{a}^{t} C(t, s) f(s) d s=\frac{c}{\theta} C(t, a)+\int_{a}^{t} C(t, s) f(s) d s \\
& -\frac{C(t, a)}{\theta} \int_{a}^{b} \varphi(w) C_{w}^{\prime}(w, a) x(a) d w \\
& -\frac{C(t, a)}{\theta} \int_{a}^{b} \varphi(w)\left(\int_{a}^{w} C_{w}^{\prime}(w, \tau) f(\tau) d \tau\right) d w \\
& -\frac{C(t, a)}{\theta} \int_{a}^{b} \varphi(w) f(w) d w .
\end{aligned}
$$

Since $C(t, s)=0, s>t$, it follows that $\int_{a}^{t} C(t, s) d s=$ $\int_{a}^{t} C(t, s) d s+\int_{t}^{b} C(t, s) d s=\int_{a}^{b} C(t, s) d s$.

Changing the order of integration in the third double integral, we get

$$
\begin{aligned}
x(t)= & \frac{c}{\theta} C(t, a)+\int_{a}^{b} C(t, w) f(w) d w \\
& -\frac{C(t, a)}{\theta} \int_{a}^{b} \varphi(w) C_{w}^{\prime}(w, a) x(a) d w \\
& -\frac{C(t, a)}{\theta} \int_{a}^{b} \int_{w}^{b} \varphi(s) C_{s}^{\prime}(s, w) f(w) d s d w \\
& -\frac{C(t, a)}{\theta} \int_{a}^{b} \varphi(w) f(w) d w \\
= & \frac{c}{\theta} C(t, a) \\
& +\int_{a}^{b}\left[C(t, w) f(w)-\frac{C(t, a)}{\theta} \varphi(w) C_{w}^{\prime}(w, a) x(a)\right. \\
& -\frac{C(t, a)}{\theta} \int_{w}^{b} \varphi(s) C_{s}^{\prime}(s, w) f(w) d s \\
= & \frac{c}{\theta} C(t, a) \\
& +\int_{a}^{b}\left[\left[C(t, w)-\frac{C(t, a)}{\theta} \varphi(w) f(w)\right] d w\right. \\
& -\int_{a}^{b} \frac{C(t, a)}{\theta} \varphi(w) C_{w}^{\prime}(w, a) x(a) d w \\
& \left.\left.\times\left(\int_{w}^{b} \varphi(s) C_{s}^{\prime}(s, w) d s+\varphi(w)\right)\right] f(w)\right] d w
\end{aligned}
$$

$$
\begin{aligned}
=\int_{a}^{b}\left[C(t, w)-\frac{C(t, a)}{\theta}\right. \\
\left.\quad \times\left(\int_{w}^{b} \varphi(s) C_{s}^{\prime}(s, w) d s+\varphi(w)\right)\right] f(w) d w \\
\quad+\frac{C(t, a)}{\theta}\left(c-x(a) \int_{a}^{b} \varphi(w) C_{w}^{\prime}(w, a) d w\right) .
\end{aligned}
$$

Substituting now (16) into (12), we obtain

$$
\begin{aligned}
& x(a)= \frac{c}{\theta}-\frac{1}{\theta} \int_{a}^{b} \varphi(s) \\
& \times\left[C_{s}^{\prime}(s, a) x(a)+\int_{a}^{s} C_{s}^{\prime}(s, w) f(w) d w+f(s)\right] d s \\
&= \frac{c}{\theta}-x(a) \frac{1}{\theta} \int_{a}^{b} \varphi(s) C_{s}^{\prime}(s, a) d s \\
&-\frac{1}{\theta} \int_{a}^{b} \varphi(s)\left[\int_{a}^{s} C_{s}^{\prime}(s, w) f(w) d w+f(s)\right] d s, \\
& x(a) {\left[\frac{1}{\theta}\left(\theta+\int_{a}^{b} \varphi(s) C_{s}^{\prime}(s, a) d s\right)\right] } \\
&= \frac{1}{\theta}\left(c-\int_{a}^{b} \varphi(s)\left[\int_{a}^{s} C_{s}^{\prime}(s, w) f(w) d w+f(s)\right] d s\right), \\
& x(a)= \frac{c-\int_{a}^{b} \varphi(s)\left[\int_{a}^{s} C_{s}^{\prime}(s, w) f(w) d w+f(s)\right] d s}{\theta+\int_{a}^{b} \varphi(s) C_{s}^{\prime}(s, a) d s} \\
&=-\frac{\int_{a}^{b} \varphi(s) f(s) d s}{\theta+\int_{a}^{b} \varphi(s) C_{s}^{\prime}(s, a) d s} \\
&+\frac{c-\int_{a}^{b} \varphi(s)\left[\int_{a}^{s} C_{s}^{\prime}(s, w) f(w) d w\right] d s}{\theta+\int_{a}^{b} \varphi(s) C_{s}^{\prime}(s, a) d s} \\
&= \frac{\int_{a}^{b} \varphi(s) f(s) d s+\int_{a}^{b} f(w) \int_{w}^{b} \varphi(s) C_{s}^{\prime}(s, w) d s d w}{\theta+\int_{a}^{b} \varphi(s) C_{s}^{\prime}(s, a) d s} \\
& \frac{c}{b} \varphi(s) C_{s}^{\prime}(s, a) d s \\
&
\end{aligned}
$$

Substituting (19) into (18), we obtain

$$
\begin{aligned}
& x(t) \\
& =\int_{a}^{b}\left[C(t, w)-\frac{C(t, a)}{\theta}\right. \\
& \left.\quad \times\left(\int_{w}^{b} \varphi(s) C_{s}^{\prime}(s, w) d s+\varphi(w)\right)\right] f(w) d w
\end{aligned}
$$




$$
\begin{aligned}
& +\frac{C(t, a)}{\theta}\left(c+\left[\left(\int_{a}^{b} \varphi(s) f(s) d s+\int_{a}^{b} f(w)\right.\right.\right. \\
& \left.\times \int_{w}^{b} \varphi(s) C_{s}^{\prime}(s, w) d s d w-c\right) \\
& \left.\times\left(\theta+\int_{a}^{b} \varphi(s) C_{s}^{\prime}(s, a) d s\right)^{-1}\right] \\
& \left.\times \int_{a}^{b} \varphi(w) C_{w}^{\prime}(w, a) d w\right) \\
& x(t)=\int_{a}^{b}\left[C(t, w)-\frac{C(t, a)}{\theta}\left(\int_{w}^{b} \varphi(s) C_{s}^{\prime}(s, w) d s+\varphi(w)\right)\right. \\
& +\frac{(C(t, a) / \theta) \int_{a}^{b} \varphi(s) C_{s}^{\prime}(s, a) d s}{\theta+\int_{a}^{b} \varphi(s) C_{s}^{\prime}(s, a) d s} \\
& \left.\times\left[\varphi(w)+\int_{w}^{b} \varphi(s) C_{s}^{\prime}(s, w) d s\right]\right] f(w) d w \\
& +\frac{C(t, a)}{\theta}\left(c-\frac{c \int_{a}^{b} \varphi(s) C_{s}^{\prime}(s, a) d s}{\theta+\int_{a}^{b} \varphi(s) C_{s}^{\prime}(s, a) d s}\right) \\
& =\int_{a}^{b} G(t, w) f(w) d w+g(t) .
\end{aligned}
$$

It is clear that Green's function $G(t, s)$ of this problem is of the following form:

$$
G(t, s)
$$

$$
\begin{aligned}
= & C(t, s)-\frac{C(t, a)}{\theta}\left(\int_{s}^{b} \varphi(\tau) C_{\tau}^{\prime}(\tau, s) d \tau+\varphi(s)\right) \\
& +\frac{(C(t, a) / \theta) \int_{a}^{b} \varphi(w) C_{w}^{\prime}(w, a) d w}{\theta+\int_{a}^{b} \varphi(w) C_{w}^{\prime}(w, a) d w} \\
& \times\left[\varphi(s)+\int_{s}^{b} \varphi(w) C_{w}^{\prime}(w, s) d w\right] \\
= & C(t, s)-\frac{C(t, a)}{\theta}\left(\int_{s}^{b} \varphi(\tau) C_{\tau}^{\prime}(\tau, s) d \tau+\varphi(s)\right) \\
& \times\left[1-\frac{\int_{a}^{b} \varphi(w) C_{w}^{\prime}(w, a) d w}{\theta+\int_{a}^{b} \varphi(w) C_{w}^{\prime}(w, a) d w}\right] \\
= & C(t, s)-C(t, a) \frac{\int_{s}^{b} \varphi(w) C_{w}^{\prime}(w, s) d w+\varphi(s)}{\theta+\int_{a}^{b} \varphi(w) C_{w}^{\prime}(w, a) d w}
\end{aligned}
$$

$$
\begin{aligned}
& \qquad \begin{aligned}
g(t) & =\frac{C(t, a)}{\theta}\left(c-\frac{c \int_{a}^{b} \varphi(s) C_{s}^{\prime}(s, a) d s}{\theta+\int_{a}^{b} \varphi(s) C_{s}^{\prime}(s, a) d s}\right) \\
& =C(t, a) \frac{c}{\theta+\int_{a}^{b} \varphi(s) C_{s}^{\prime}(s, a) d s},
\end{aligned} \\
& \text { and } C(t, s)=0, \text { for } t<s .
\end{aligned}
$$

One has

$$
x(t)=\int_{a}^{b} G(t, s) f(s) d s+g(t) .
$$

Theorem 1 has been proven.

Theorem 1 demonstrates the importance to know exactly or approximately the Cauchy function $C(t, s)$ of impulsive equations (1)-(4).

Substituting $c=0, \varphi(s) \equiv \gamma, \theta=\gamma-1$ we obtain the Corollary.

Corollary 2. If generalized periodic problem (1)-(4), (7) is uniquely solvable, then its solution can be represented in the following form:

$$
x(t)=\int_{a}^{b} W(t, s) f(s) d s,
$$

where the Green's function $W(t, s)$ is as follows:

$$
W(t, s)=C(t, s)-C(t, a) \frac{\gamma \int_{s}^{b} C_{w}^{\prime}(w, s) d w+\gamma}{(\gamma-1)+\gamma \int_{a}^{b} C_{w}^{\prime}(w, a) d w} .
$$

\section{Auxiliary Results}

Let us construct the Cauchy function $C(t, s)$ for several simple equations.

Consider now the following auxiliary equation:

$$
x^{\prime}(t)+p(t) x(t)=f(t) .
$$

Let us denote the Cauchy function of the nonimpulsive equation (25) by $K(t, s)$. It is known that $K(t, s)=e^{-\int_{s}^{t} p(\zeta) d \zeta}$. For every $s$ this function is absolutely continuous function with respect to $t$, and $K(t, s)=0$ for $s>t$.

Using the fact that the Cauchy function $C(t, s)$ for every fixed $s$ as a function of $t$ is a solution of

$$
x^{\prime}(t)+p(t) x(t)=0, \quad t \in[s, b]
$$

satisfying the condition $C(s, s)=1$, we obtain the following theorem. 


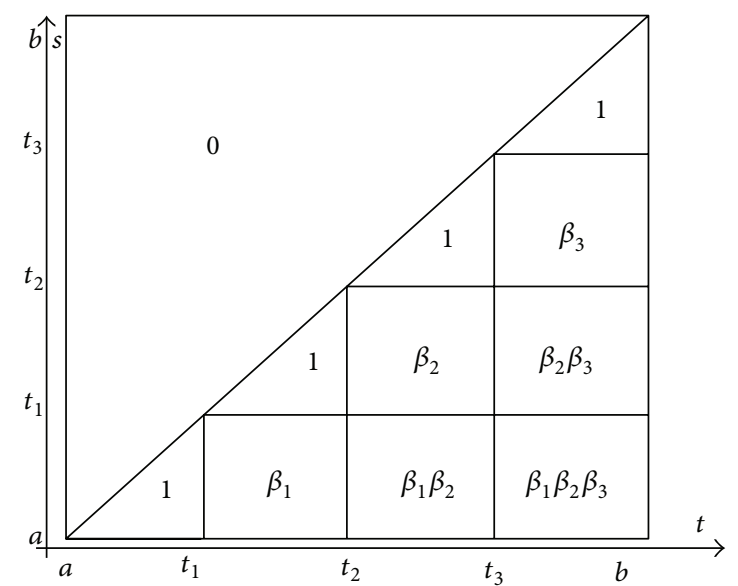

FIGURE 1

Theorem 3. The Cauchy function $C(t, s)$ of (25) and (2) can be represented in the following form:

$$
\begin{gathered}
C(t, s)=\sum_{i=1}^{k} \sum_{j=0}^{i-1} \prod_{r=j+1}^{i} \beta_{r} K(t, s)\left[H_{t_{i}}(t)-H_{t_{i+1}}(t)\right] \\
\times\left[H_{t_{j}}(s)-H_{t_{j+1}}(s)\right] \\
+\sum_{i=0}^{k} H_{0}(t-s) K(t, s)\left[H_{t_{i}}(t)-H_{t_{i+1}}(t)\right] \\
\times\left[H_{t_{i}}(s)-H_{t_{i+1}}(s)\right],
\end{gathered}
$$

where $H_{a}(t)$, Heaviside function, is defined by the following equality:

$$
H_{a}(t)= \begin{cases}1, & t \geq a, \\ 0, & t<a .\end{cases}
$$

Now let us consider the following auxiliary equation:

$$
\begin{gathered}
x^{\prime}(t)=f(t), \quad t \in[a, b], \\
x\left(t_{j}\right)=\beta_{j} x\left(t_{j}-0\right), \quad j=1, \ldots, k, \\
a=t_{0}<t_{1}<t_{2}<\cdots<t_{k}<t_{k+1}=b .
\end{gathered}
$$

Figure 1 describes the Cauchy function $C_{0}(t, s)$ of the problem (29), (2), and (4) in the case $k=3$.

In the case of $k$ impulses we obtain the following theorem.

Theorem 4. The Cauchy function $C_{0}(t, s)$ of the problem (29), (2) and its derivatives has the following representations:

$$
\begin{aligned}
C_{0}(t, s)=\sum_{i=1}^{k} \sum_{j=0}^{i-1} \prod_{r=j+1}^{i} \beta_{r} & {\left[H_{t_{i}}(t)-H_{t_{i+1}}(t)\right] } \\
\times & {\left[H_{t_{j}}(s)-H_{t_{j+1}}(s)\right] }
\end{aligned}
$$

$$
\begin{aligned}
& +\sum_{i=0}^{k} H_{0}(t-s)\left[H_{t_{i}}(t)-H_{t_{i+1}}(t)\right] \\
& \times\left[H_{t_{i}}(s)-H_{t_{i+1}}(s)\right], \\
& C_{0}(t, a)=\sum_{i=1}^{k} \prod_{r=1}^{i} \beta_{r}\left[H_{t_{i}}(t)-H_{t_{i+1}}(t)\right] \\
& +H_{0}(t-a)\left[H_{t_{0}}(t)-H_{t_{1}}(t)\right], \\
& \left(C_{0}(t, s)\right)_{t}^{\prime}=\sum_{i=1}^{k} \sum_{j=0}^{i-1} \prod_{r=j+1}^{i} \beta_{r}\left[H_{t_{j}}(s)-H_{t_{j+1}}(s)\right] \\
& \times\left[\delta_{t_{i}}(t)-\delta_{t_{i+1}}(t)\right] \\
& +\sum_{i=0}^{k}\left[H_{t_{i}}(s)-H_{t_{i+1}}(s)\right] \\
& \times\left(\delta_{0}(t-s)\left[H_{t_{i}}(t)-H_{t_{i+1}}(t)\right]\right. \\
& \left.+H_{0}(t-s)\left[\delta_{t_{i}}(t)-\delta_{t_{i+1}}(t)\right]\right), \\
& \left(C_{0}(t, a)\right)_{t}^{\prime} \\
& =\sum_{i=1}^{k} \prod_{r=1}^{i} \beta_{r}\left[\delta_{t_{i}}(t)-\delta_{t_{i+1}}(t)\right] \\
& +\delta_{0}(t-a)\left[H_{t_{0}}(t)-H_{t_{1}}(t)\right] \\
& +H_{0}(t-a)\left[\delta_{t_{0}}(t)-\delta_{t_{1}}(t)\right] .
\end{aligned}
$$

Denote by $G_{0}(t, s)$ Green's function of problem (29), (5), and by $W_{0}(t, s)$ Green's function of generalized periodic problem (29), (7).

Let us denote $I(\varphi, \theta, \beta)=\theta+\int_{a}^{b} \varphi(t)\left(C_{0}(t, a)\right)_{t}^{\prime} d t$.

Let $\beta=\operatorname{Col}\left\{\beta_{1}, \ldots, \beta_{k}\right\}$. The computation of integrals in formula (11) leads us to the following equality:

$I(\varphi, \theta, \beta)$

$$
=\theta+\sum_{i=1}^{k-1} \prod_{r=1}^{i} \beta_{r}
$$

$$
\begin{aligned}
& \times\left[\frac{\varphi\left(t_{i}+\right)+\varphi\left(t_{i}-\right)}{2}-\frac{\varphi\left(t_{i+1}+\right)+\varphi\left(t_{i+1}-\right)}{2}\right] \\
& +\beta_{1} \beta_{2} \cdots \beta_{k}\left[\frac{\varphi\left(t_{k}+\right)+\varphi\left(t_{k}-\right)}{2}-\frac{\varphi\left(t_{k+1}-\right)}{2}\right] \\
& +\frac{\varphi\left(t_{0}+\right)}{2}-\frac{\varphi\left(t_{1}+\right)+\varphi\left(t_{1}-\right)}{2}+\frac{\varphi\left(t_{0}+\right)}{2} .
\end{aligned}
$$


Denoting $I_{1}(\varphi, \beta)=\varphi(s)+\int_{s}^{b} \varphi(t)\left(C_{0}(t, s)\right)_{t}^{\prime} d t$, we obtain for $a \leq s<t_{1}$

$$
\begin{aligned}
& I_{1}(\varphi, \beta) \\
& =\varphi(s) \\
& +\sum_{i=1}^{k-1} \prod_{r=1}^{i} \beta_{r} \\
& \quad \times\left[\frac{\varphi\left(t_{i}+\right)+\varphi\left(t_{i}-\right)}{2}-\frac{\varphi\left(t_{i+1}+\right)+\varphi\left(t_{i+1}-\right)}{2}\right] \\
& \quad+\beta_{1} \beta_{2} \cdots \beta_{k}\left[\frac{\varphi\left(t_{k}+\right)+\varphi\left(t_{k}-\right)}{2}-\frac{\varphi\left(t_{k+1}-\right)}{2}\right] \\
& \quad+\frac{\varphi(s+)}{2}+H_{0}\left(t_{0}-s\right) \frac{\varphi\left(t_{0}+\right)}{2}-\frac{\varphi\left(t_{1}+\right)+\varphi\left(t_{1}-\right)}{2} .
\end{aligned}
$$

Denoting $I_{2}(\varphi, \beta)=\varphi(s)+\int_{s}^{b} \varphi(t)\left(C_{0}(t, s)\right)_{t}^{\prime} d t$, we obtain for $t_{1} \leq s<t_{2}$

$$
\begin{aligned}
& I_{2}(\varphi, \beta) \\
& =\varphi(s) \\
& +\sum_{i=2}^{k-1} \prod_{r=2}^{i} \beta_{r} \\
& \quad \times\left[\frac{\varphi\left(t_{i}+\right)+\varphi\left(t_{i}-\right)}{2}-\frac{\varphi\left(t_{i+1}+\right)+\varphi\left(t_{i+1}-\right)}{2}\right] \\
& \quad+\beta_{2} \beta_{3} \cdots \beta_{k}\left[\frac{\varphi\left(t_{k}+\right)+\varphi\left(t_{k}-\right)}{2}-\frac{\varphi\left(t_{k+1}-\right)}{2}\right] \\
& \quad+\frac{\varphi(s+)}{2}+H_{0}\left(t_{1}-s\right) \frac{\varphi\left(t_{1}+\right)}{2}-\frac{\varphi\left(t_{2}+\right)+\varphi\left(t_{2}-\right)}{2}
\end{aligned}
$$

Denoting $I_{k+1}(\varphi, \beta)=\varphi(s)+\int_{s}^{b} \varphi(t)\left(C_{0}(t, s)\right)_{t}^{\prime} d t$, we obtain for $t_{k} \leq s<b$

$$
\begin{aligned}
I_{k+1}(\varphi, \beta)= & \varphi(s)+\frac{\varphi(s+)}{2} \\
& +H_{0}\left(t_{k}-s\right) \frac{\varphi\left(t_{k}+\right)}{2}-\frac{\varphi\left(t_{k+1}-\right)}{2} .
\end{aligned}
$$

Let us denote $J(\beta, \gamma)=(\gamma-1)+\gamma \int_{a}^{b}\left(C_{0}(t, a)\right)_{t}^{\prime} d t$.

The computation of integrals in formula (24) leads us to the following equality:

$$
\begin{aligned}
J(\beta, \gamma)= & (\gamma-1) \\
& +\gamma\left(\sum_{i=1}^{k} \prod_{r=1}^{i} \beta_{r}\left[H_{t_{i}}(t)-H_{t_{i+1}}(t)\right]+1\right) .
\end{aligned}
$$

Denoting $J_{1}(\beta, \gamma)=\gamma \int_{s}^{b}\left(C_{0}(t, s)\right)_{t}^{\prime} d t+\gamma$, we obtain for $a \leq s<t_{1}$

$$
\begin{aligned}
J_{1}(\beta, \gamma)=\gamma\left(\sum_{i=1}^{k} \prod_{r=1}^{i} \beta_{r}\left[H_{t_{i}}(t)-H_{t_{i+1}}(t)\right]\right. \\
\left.\quad+H_{t_{0}}(s)+H_{0}\left(t_{0}-s\right)-1\right)+\gamma .
\end{aligned}
$$

Denoting $J_{2}(\beta, \gamma)=\gamma \int_{s}^{b}\left(C_{0}(t, s)\right)_{t}^{\prime} d t+\gamma$, we obtain for $t_{1} \leq s<t_{2}$

$$
\begin{array}{r}
J_{2}(\beta, \gamma)=\gamma\left(\sum_{i=2}^{k} \prod_{r=1}^{i} \beta_{r}\left[H_{t_{i}}(t)-H_{t_{i+1}}(t)\right]\right. \\
\left.+H_{t_{1}}(s)+H_{0}\left(t_{1}-s\right)\right)+\gamma
\end{array}
$$

Denoting $J_{k+1}(\beta)=\gamma \int_{s}^{b}\left(C_{0}(t, s)\right)_{t}^{\prime} d t+\gamma$, we obtain for $t_{k} \leq s<b$

$$
\begin{gathered}
J_{k+1}(\beta)=\gamma\left(H_{t_{k}}(s)-H_{t_{k+1}}(s)+H_{0}\left(t_{k}-s\right)\right. \\
\left.-H_{0}\left(t_{k+1}-s\right)\right)+\gamma \\
=\gamma\left(H_{t_{k}}(s)+H_{0}\left(t_{k}-s\right)\right)+\gamma .
\end{gathered}
$$

Corollary 5. Let us assume that $I(\varphi, \theta, \beta) \neq 0$; then Green's function $G_{0}(t, s)$ of problem (29), (5) in the case of three impulsive points (i.e., $k=3$ ) is described by Figure 2.

Corollary 6. Let us assume that $J(\beta, \gamma) \neq 0$; then Green's function $W_{0}(t, s)$ of generalized periodic problem (29), (7) in the case of three impulsive points (i.e. $k=3$ ) is described by Figure 3.

\section{Estimation of Solutions}

Let us denote $\beta=\max _{1 \leq i \leq k} \beta_{i}, \Omega=(1 /|I(\varphi, \theta, \beta)|) \mid\left(1-\beta^{k+1}\right) /$ $(1-\beta) \mid((2 k+1) / 2) \Delta$ where $\Delta=\left(\max _{1 \leq i \leq k+1}\left|t_{i}-t_{i-1}\right|\right)^{2}$,

$$
\begin{aligned}
\widetilde{\Omega}=\Omega & {\left[|\theta|+\|\varphi\|\left(5+4 \sum_{i=1}^{k} \beta^{i}\right)\right.} \\
& \left.+\left(\max _{1 \leq i \leq k+1} \beta^{i-1}\right)\|\varphi\|\left(3+2 \sum_{i=1}^{k} \beta^{i}\right)\right] .
\end{aligned}
$$

Theorem 7. Let $0<\beta, 1 \leq i \leq k, I(\varphi, \theta, \beta) \neq 0$ and

$$
m(b-a) \text { ess } \sup _{\substack{s \in[a, b] \\ 1 \leq i \leq n}}\left|p_{i}(s)\right| \widetilde{\Omega}<1
$$




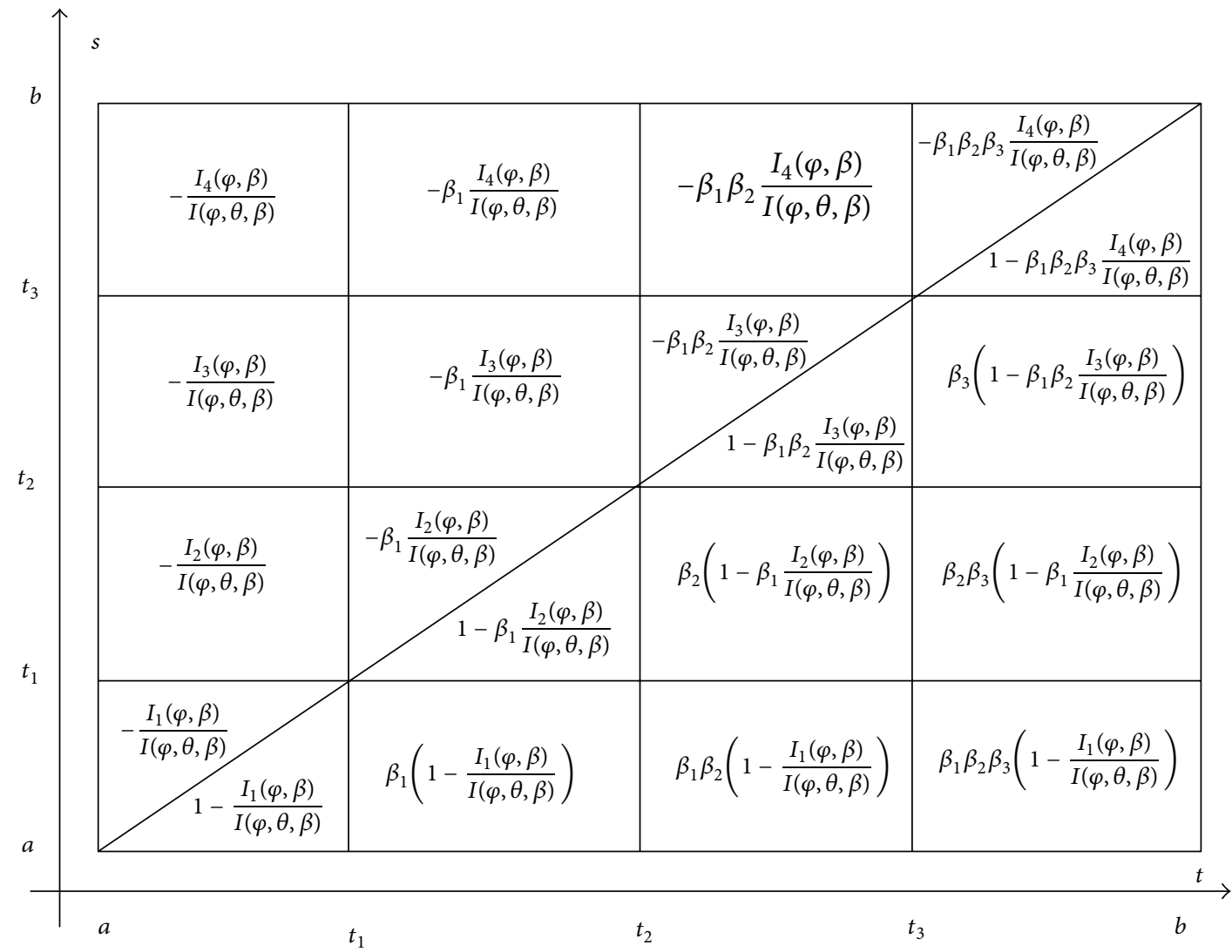

Figure 2

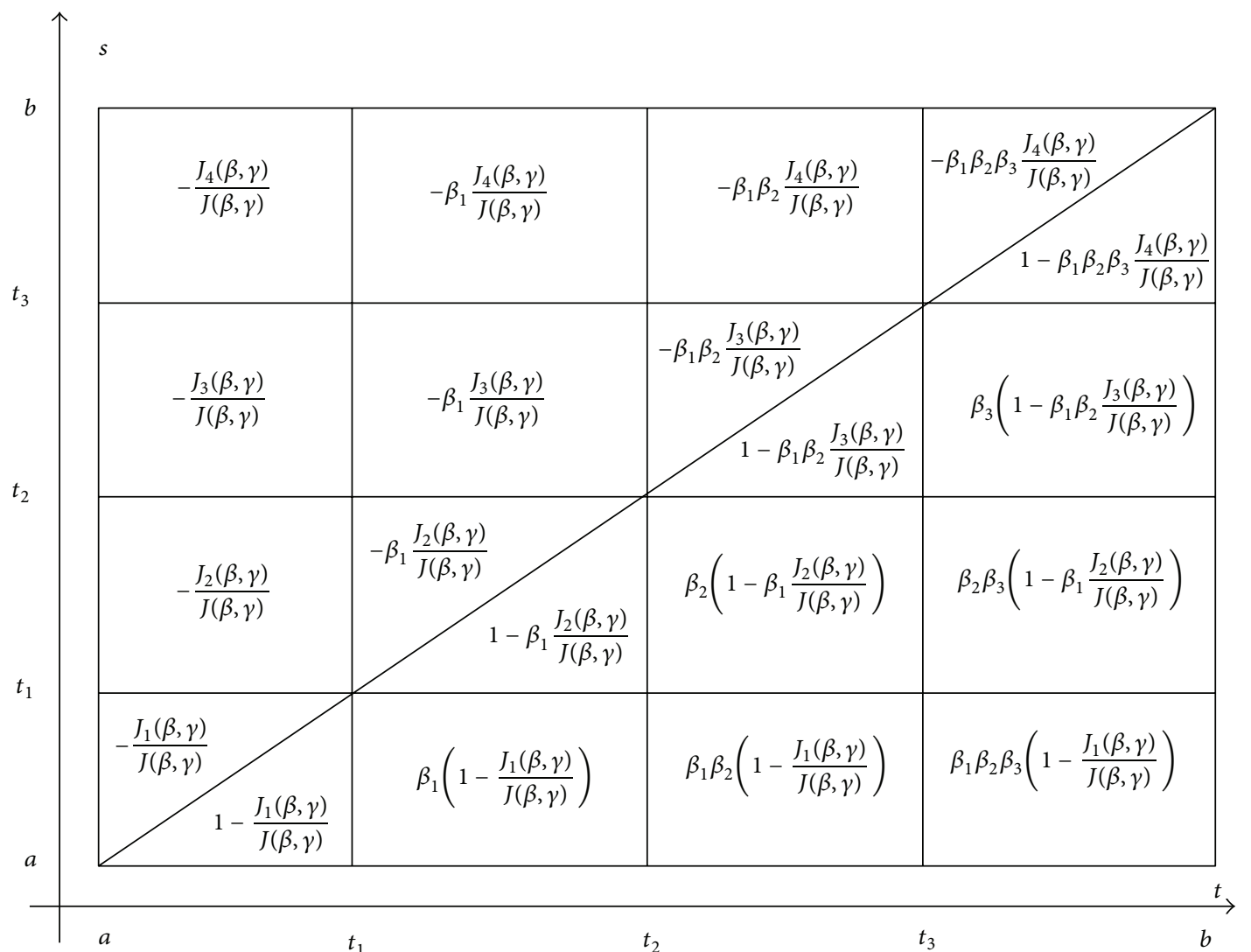

Figure 3 
then there exists a unique solution of problem (1)-(5) and this solution can be estimated as follows:

$\|x\|$

$$
\leq \frac{\widetilde{\Omega}(b-a)\|f\||I(\varphi, \theta, \beta)|+|c| \max \left[\max _{1 \leq j \leq k} \prod_{i=1}^{j} \beta_{i}, 1\right]}{(1-\widetilde{\Omega})|I(\varphi, \theta, \beta)|},
$$

for every essentially bounded function $f$ and real c.

Proof. By Theorem 1, problem (1)-(5) is equivalent to the integral equation:

$$
\begin{aligned}
x(t)= & \int_{a}^{b} G_{0}(t, s) \phi(s) d s \\
& +C_{0}(t, a) \frac{c}{\theta+\int_{a}^{b} \varphi(s)\left(C_{0}(s, a)\right)_{s}^{\prime} d s} \\
= & \int_{a}^{b} G_{0}(t, s) f(s) d s \\
& +C_{0}(t, a) \frac{c}{\theta+\int_{a}^{b} \varphi(s)\left(C_{0}(s, a)\right)_{s}^{\prime} d s} \\
& -\int_{a}^{b} G_{0}(t, s) \sum_{i=1}^{m} p_{i}(s) x\left(s-\tau_{i}(s)\right) d s .
\end{aligned}
$$

Let us denote $\psi(t)=\int_{a}^{b} G_{0}(t, s) f(s) d s+C_{0}(t, a)(c /(\theta+$ $\left.\left.\int_{a}^{b} \varphi(s)\left(C_{0}(s, a)\right)_{s}^{\prime} d s\right)\right)=\int_{a}^{b} G_{0}(t, s) f(s) d s+\left(c C_{0}(t, a) / I(\varphi, \theta\right.$, $\beta)$.

Define the operator $M: D\left(t_{1}, \ldots, t_{k}\right) \rightarrow D\left(t_{1}, \ldots, t_{k}\right)$ by the equality $(M x)(t)=-\int_{a}^{b} G_{0}(t, s) \sum_{i=1}^{m} p_{i}(s) x\left(s-\tau_{i}(s)\right) d s$. (5):

We can write equation which is equivalent to problem (1)-

$$
x(t)=(M x)(t)+\psi(t) .
$$

If $\|M\|<1$, then there exists the unique solution of (1)-(5) which can be represented in the form: $x(t)=(I-$ $M)^{-1} \psi(t)$.

It is clear that

$$
\begin{aligned}
\|M x\| \leq & \left\|G_{0}\right\| \sum_{i=1}^{m} \int_{a}^{b}\left|p_{i}(s)\right|\left|x\left(s-\tau_{i}(s)\right)\right| \chi_{[a, b]} \\
& \times\left(s-\tau_{i}(s)\right) d s \\
\leq & \left\|G_{0}\right\| m(b-a) \underset{\substack{s \in[a, b] \\
1 \leq i \leq m}}{\operatorname{ess} \sup }\left|p_{i}(s)\right|\|x\|,
\end{aligned}
$$

where $\|x\|=\max _{a \leq t \leq b}|x(t)|$. Thus we have got the following condition for existence of unique solution of problem (1)-(5):

$$
\|M\| \leq\left\|G_{0}\right\| m(b-a) \text { ess } \sup _{\substack{s \in[a, b] \\ 1 \leq i \leq n}}\left|p_{i}(s)\right|<1 .
$$

\section{Denote}

$$
g(t)=\frac{c C_{0}(t, a)}{I(\varphi, \theta, \beta)}
$$

where $C_{0}(t, a)$ can be written as

$$
C_{0}(t, a)= \begin{cases}1, & a \leq t<t_{1}, \\ \beta_{1}, & t_{1} \leq t<t_{2}, \\ \beta_{1} \beta_{2}, & t_{2} \leq t<t_{3}, \\ \vdots & \\ \beta_{1} \cdot \ldots \cdot \beta_{k}, & t_{k} \leq t<b .\end{cases}
$$

It is clear that

$$
\|g\| \leq \frac{|c| \max \left[\max _{1 \leq j \leq k} \prod_{i=1}^{j} \beta_{i}, 1\right]}{|I(\varphi, \theta, \beta)|} .
$$

Estimation of the solution of the problem (1)-(5) can be done as follows:

$$
\begin{aligned}
& |x(t)| \leq\left\|G_{0}\right\| \int_{a}^{b}|f(s)| d s+\|g\|+\|M x\| \\
& \Longrightarrow\|x\| \\
& \leq\left\|G_{0}\right\|(b-a) \underset{t \in[a, b]}{\operatorname{ess} \sup }|f(t)| \\
& +\|g\|+\left\|G_{0}\right\| m(b-a) \text { ess } \sup _{\substack{s \in[a, b] \\
1 \leq i \leq n}}\left|p_{i}(s)\right|\|x\|
\end{aligned}
$$

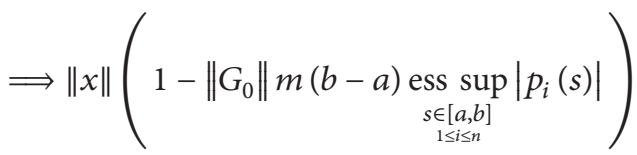

$$
\begin{aligned}
& \leq\left\|G_{0}\right\|(b-a)\|f\|+\|g\| .
\end{aligned}
$$

Let us denote

$$
Q=\left\|G_{0}\right\| m(b-a) \underset{\substack{\text { ess } \\ s \in[a, b] \\ 1 \leq i \leq n}}{ }\left|p_{i}(s)\right|
$$

Then the problem (1)-(5) has unique solution if

$$
\|M\| \leq Q<1 .
$$

It is clear that

$$
\|x\| \leq \frac{\left\|G_{0}\right\|(b-a)\|f\|+\|g\|}{1-Q} .
$$


Estimating the integrals in formula (11), we get the following inequalities:

$$
\begin{gathered}
|I(\varphi, \theta, \beta)| \leq|\theta|+2\|\varphi\|+2\|\varphi\| \sum_{i=1}^{k} \beta^{i} \\
=|\theta|+\|\varphi\|\left(2+2 \sum_{i=1}^{k} \beta^{i}\right), \\
\left|I_{1}(\varphi, \beta)\right| \leq\|\varphi\|+2\|\varphi\| \sum_{i=1}^{k} \beta^{i}+2\|\varphi\| \\
=3\|\varphi\|+2\|\varphi\| \sum_{i=1}^{k} \beta^{i}=\|\varphi\|\left(3+2 \sum_{i=1}^{k} \beta^{i}\right) \\
\left|I_{2}(\varphi, \beta)\right| \leq\|\varphi\|+2\|\varphi\| \sum_{i=2}^{k} \beta^{i}+2\|\varphi\| \\
=3\|\varphi\|+2\|\varphi\| \sum_{i=2}^{k} \beta^{i}=\|\varphi\|\left(3+2 \sum_{i=2}^{k} \beta^{i}\right) \\
\vdots \\
\left|I_{k+1}(\varphi, \beta)\right| \leq 2\|\varphi\| .
\end{gathered}
$$

Let us write $G_{0}=G_{1}+G_{2}$, where $G_{1}$ is upper triangular and $G_{2}$ lower triangular parts of $G_{0}$ and estimate $\left\|G_{0}\right\|$. One has

$$
\begin{aligned}
\left\|G_{0}\right\| \leq & \left\|G_{1}\right\|+\left\|G_{2}\right\| \\
\leq \Omega & {\left[\max _{1 \leq i \leq k+1}\left|I_{i}(\varphi, \beta)\right|\right.} \\
& \left.\quad+\max _{1 \leq i \leq k+1}\left|I(\varphi, \theta, \beta)-\beta^{i-1} I_{i}(\varphi, \beta)\right|\right] \\
\leq \Omega & {\left[\max _{1 \leq i \leq k+1}\left|I_{i}(\varphi, \beta)\right|+|I(\varphi, \theta, \beta)|\right.} \\
& \left.\quad+\max _{1 \leq i \leq k+1}\left|I_{i}(\varphi, \beta)\right| \beta^{i-1}\right] \\
=\Omega & {\left[|I(\varphi, \theta, \beta)|+\max _{1 \leq i \leq k+1}\left|I_{i}(\varphi, \beta)\right|\left(\beta^{i-1}+1\right)\right] . }
\end{aligned}
$$

From estimation of the integrals, we obtain

$$
\begin{aligned}
\left\|G_{0}\right\| \leq \Omega & {\left[|\theta|+\|\varphi\|\left(2+2 \sum_{i=1}^{k} \beta^{i}\right)\right.} \\
& \left.+\left(1+\max _{1 \leq i \leq k+1} \beta^{i-1}\right)\|\varphi\|\left(3+2 \sum_{i=1}^{k} \beta^{i}\right)\right]
\end{aligned}
$$

$$
\begin{aligned}
& \leq \Omega\left[|\theta|+\|\varphi\|\left(5+4 \sum_{i=1}^{k} \beta^{i}\right)\right. \\
& \left.\quad+\left(\max _{1 \leq i \leq k+1} \beta^{i-1}\right)\|\varphi\|\left(3+2 \sum_{i=1}^{k} \beta^{i}\right)\right]=\widetilde{\Omega} .
\end{aligned}
$$

By (50)

$$
\begin{aligned}
Q \leq & m(b-a) \operatorname{ess} \sup _{\substack{s \in[a, b] \\
1 \leq i \leq n}}\left|p_{i}(s)\right| \Omega \\
\times & {\left[|\theta|+\|\varphi\|\left(5+4 \sum_{i=1}^{k} \beta^{i}\right)\right.} \\
& \left.+\left(\max _{1 \leq i \leq k+1} \beta^{i-1}\right)\|\varphi\|\left(3+2 \sum_{i=1}^{k} \beta^{i}\right)\right] .
\end{aligned}
$$

Substituting (56) into (51) and (52) we obtain

$$
\begin{aligned}
\|M\| \leq & m(b-a) \text { ess } \sup _{\substack{s \in[a, b] \\
1 \leq i \leq n}}\left|p_{i}(s)\right| \Omega \\
\times & {\left[|\theta|+\|\varphi\|\left(5+4 \sum_{i=1}^{k} \beta^{i}\right)\right.} \\
& \left.+\left(\max _{1 \leq i \leq k+1} \beta^{i-1}\right)\|\varphi\|\left(3+2 \sum_{i=1}^{k} \beta^{i}\right)\right] \\
\|x\| \leq & (\widetilde{\Omega}(b-a)\|f\| \\
& \left.+\frac{|c| \max \left[\max _{1 \leq j \leq k} \prod_{i=1}^{j} \beta_{i}, 1\right]}{|I(\varphi, \theta, \beta)|}\right)(1-\widetilde{\Omega})^{-1} \\
= & (\widetilde{\Omega}(b-a)\|f\||I(\varphi, \theta, \beta)| \\
& \left.+|c| \max \left[\max _{1 \leq j \leq k} \prod_{i=1}^{j} \beta_{i}, 1\right]\right)((1-\widetilde{\Omega})|I(\varphi, \theta, \beta)|)^{-1} .
\end{aligned}
$$

Theorem 7 has been proven.

Let us denote $\Omega_{2}=\left|1-\beta^{k+1}\right|(2 k+1) \Delta / 2|1-\beta||J(\beta, \gamma)|$, $\widetilde{\Omega_{2}}$

$$
=\Omega_{2}\left[|\gamma|\left(5+2 \sum_{i=1}^{k} \beta^{i}\right)+\max _{1 \leq i \leq k+1} \beta^{i-1}|\gamma|\left(3+\sum_{i=1}^{k} \beta^{i}\right)\right] .
$$

Theorem 8. Let $0<\beta_{i}, 1 \leq i \leq k, J(\beta, \gamma) \neq 0$ and

$$
\widetilde{\Omega_{2}} m(b-a) \text { ess } \sup _{\substack{s \in[a, b] \\ 1 \leq i \leq m}}\left|p_{i}(s)\right|<1 ;
$$


then there exists a unique solution of the generalized periodic problem (1)-(4), (7) and this solution can be estimated as follows:

$$
\|x\| \leq \frac{\widetilde{\Omega_{2}}(b-a)\|f\|}{1-\widetilde{\Omega_{2}} m(b-a) \text { ess } \sup _{\substack{s \in[a, b] \\ 1 \leq i \leq m}}\left|p_{i}(s)\right|}
$$

for every essentially bounded function $f$.

Proof. By Corollary 2, problem (1)-(4), (7) is equivalent to the following integral equation:

$$
\begin{aligned}
x(t)= & \int_{a}^{b} W_{0}(t, s) \phi(s) d s \\
= & \int_{a}^{b} W_{0}(t, s) f(s) d s \\
& -\int_{a}^{b} W_{0}(t, s) \sum_{i=1}^{m} p_{i}(s) x\left(s-\tau_{i}(s)\right) d s .
\end{aligned}
$$

Let us denote $\psi(t)=\int_{a}^{b} W_{0}(t, s) f(s) d s$.

Define the operator $T: D\left(t_{1}, \ldots, t_{k}\right) \rightarrow D\left(t_{1}, \ldots, t_{k}\right)$ by the equality $(T x)(t)=-\int_{a}^{b} W_{0}(t, s) \sum_{i=1}^{m} p_{i}(s) x\left(s-\tau_{i}(s)\right) d s$.

We can write equation which is equivalent to problem (1)(4), (7) as follows:

$$
x(t)=(T x)(t)+\psi(t) .
$$

If $\|T\|<1$, then there exists the unique solution of (1)$(4),(7)$ which can be represented in the form: $x(t)=(I-$ $M)^{-1} \psi(t)$.

It is clear that

$$
\begin{aligned}
\|T x\| \leq\left\|W_{0}\right\| \sum_{i=1}^{m} \int_{a}^{b}\left|p_{i}(s)\right|\left|x\left(s-\tau_{i}(s)\right)\right| \chi_{[a, b]} \\
\quad \times\left(s-\tau_{i}(s)\right) d s \\
\leq\left\|W_{0}\right\| m(b-a) \underset{\substack{\operatorname{ess} \sup _{s \in[a, b]} \mid \\
1 \leq i \leq m}}{ }\left|p_{i}(s)\right|\|x\|,
\end{aligned}
$$

where $\|x\|=\max _{a \leq t \leq b}|x(t)|$. Thus we have got the following condition for existence of unique solution of problem (1)-(4), (7):

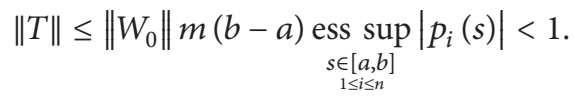

Estimation of the solution of the problem (1)-(4), (7) can be done as follows:

$$
\begin{aligned}
& |x(t)| \leq\left\|W_{0}\right\| \int_{a}^{b}|f(s)| d s+\|T x\| \\
& \Longrightarrow\|x\| \\
& \leq\left\|W_{0}\right\|(b-a) \operatorname{ess} \sup _{t \in[a, b]}|f(t)| \\
& +\left\|W_{0}\right\| m(b-a) \operatorname{ess} \sup _{\substack{s \in[a, b] \\
1 \leq i \leq m}}\left|p_{i}(s)\right|\|x\| \\
& \Longrightarrow\|x\|\left(1-\left\|W_{0}\right\| m(b-a) \operatorname{ess} \sup _{\substack{s \in[a, b] \\
1 \leq i \leq m}}\left|p_{i}(s)\right|\right) \\
& \leq\left\|W_{0}\right\|(b-a)\|f\| .
\end{aligned}
$$

Let us denote

$$
Q=\left\|W_{0}\right\| m(b-a) \text { ess } \sup _{\substack{s \in[a, b] \\ 1 \leq i \leq m}}\left|p_{i}(s)\right| .
$$

Then the problem (1)-(4), (7) has unique solution if

$$
\|T\| \leq Q<1 .
$$

It is clear that

$$
\|x\| \leq \frac{\left\|W_{0}\right\|(b-a)\|f\|}{1-Q} .
$$

Estimating the integrals in formula (24), we get the following inequalities:

$$
\begin{gathered}
|J(\beta, \gamma)| \leq|\gamma|+|\gamma|\left(1+\sum_{i=1}^{k} \beta^{i}\right)=|\gamma|\left(2+\sum_{i=1}^{k} \beta^{i}\right), \\
\left|J_{1}(\beta, \gamma)\right| \leq|\gamma|\left(\sum_{i=1}^{k} \beta^{i}+1\right)+|\gamma| \leq|\gamma|\left(3+\sum_{i=1}^{k} \beta^{i}\right), \\
\left|J_{2}(\beta, \gamma)\right| \leq|\gamma|\left(\sum_{i=2}^{k} \beta^{i}+2\right)+|\gamma| \leq|\gamma|\left(3+\sum_{i=2}^{k} \beta^{i}\right), \\
\vdots \\
\sup _{1 \leq i \leq k+1}\left|J_{i}(\beta, \gamma)\right| \leq|\gamma|\left(3+\sum_{i=1}^{k} \beta^{i}\right) .
\end{gathered}
$$


Let us write $W_{0}=W_{1}+W_{2}$, where $W_{1}$ is upper triangular and $W_{2}$, lower triangular parts, and estimate $\left\|W_{0}\right\|$ :

$$
\begin{aligned}
\left\|W_{0}\right\| \leq & \left\|W_{1}\right\|+\left\|W_{2}\right\| \\
\leq \Omega_{2}\left[\max _{1 \leq i \leq k+1}\left|J_{i}(\beta, \gamma)\right|\right. & \\
& \left.+\max _{1 \leq i \leq k+1}\left|J(\beta, \gamma)-\beta^{i-1} J_{i}(\beta, \gamma)\right|\right] \\
\leq \Omega_{2}\left[\max _{1 \leq i \leq k+1}\left|J_{i}(\beta, \gamma)\right|+|J(\beta, \gamma)|\right. & \left.\quad+\max _{1 \leq i \leq k+1}\left|J_{i}(\beta, \gamma)\right| \beta^{i-1}\right] \\
\leq & \Omega_{2}\left[|J(\beta, \gamma)|+\max _{1 \leq i \leq k+1}\left|J_{i}(\beta, \gamma)\right|\left(\beta^{i-1}+1\right)\right] .
\end{aligned}
$$

We obtain

$$
\begin{gathered}
\left\|W_{0}\right\| \leq \Omega_{2}\left[|\gamma|\left(2+\sum_{i=1}^{k} \beta^{i}\right)+\left(1+\max _{1 \leq i \leq k+1} \beta^{i-1}\right)\right. \\
\left.\times|\gamma|\left(3+\sum_{i=1}^{k} \beta^{i}\right)\right] .
\end{gathered}
$$

It is clear that

$$
\begin{aligned}
\|T\| \leq & Q \\
\leq & \Omega_{2}\left[|\gamma|\left(5+2 \sum_{i=1}^{k} \beta^{i}\right)\right. \\
& \left.\quad+\max _{1 \leq i \leq k+1} \beta^{i-1}|\gamma|\left(3+\sum_{i=1}^{k} \beta^{i}\right)\right] \\
& \times m(b-a) \underset{\substack{s \in[a, b] \\
1 \leq i \leq m}}{\operatorname{ess} \sup }\left|p_{i}(s)\right| \\
= & \widetilde{\Omega_{2}} m(b-a) \sup _{\substack{s \in[a, b] \\
1 \leq i \leq m}}\left|p_{i}(s)\right| .
\end{aligned}
$$

We obtain the following solution estimation:

$$
\|x\| \leq \frac{\widetilde{\Omega_{2}}(b-a)\|f\|}{1-\widetilde{\Omega_{2}} m(b-a) \text { ess } \sup _{\substack{s \in[a, b] \\ 1 \leq i \leq m}}\left|p_{i}(s)\right|} .
$$

Theorem 8 has been proven.

\section{Sign Constancy of Green's Functions}

The proofs of the following two assertions follow from the construction of Green's functions.
Theorem 9. Let

$$
0<\beta_{i}, \quad 1 \leq i \leq k
$$

$$
I(\varphi, \theta, \beta) \cdot I_{j}(\varphi, \theta, \beta)<0, \quad j=1, \ldots, k+1 ;
$$

then Green's function $G_{0}(t, s)$ of problem (29), (5) is positive for $t, s \in[a, b]$.

Theorem 10. Let

$$
\begin{array}{ll}
0<\beta_{i}, \quad 1 \leq i \leq k, & \frac{I_{1}(\varphi, \theta, \beta)}{I(\varphi, \theta, \beta)}>1, \\
\frac{I_{j}(\varphi, \theta, \beta)}{I(\varphi, \theta, \beta)}>\frac{1}{\prod_{i=1}^{j-1} \beta_{i}} \quad 2 \leq j \leq k+1 ;
\end{array}
$$

then Green's function $G_{0}(t, s)$ of problem (29), (5) is negative for $t, s \in[a, b]$.

Estimation of Green's function (11) leads us to the assertion.

Theorem 11. Let conditions (40) and (75) be fulfilled, $p_{i}(t) \leq 0$ for $i=1, \ldots, m$; then problem (1)-(5) is unique solvable and its Green's function $G(t, s)$ is positive for $t, s \in[a, b]$.

Proof. Without losing generality we assume that $c=0$.

Solution of problem (1)-(5) can be represented in the following form:

$$
x(t)=(M x)(t)+\psi(t),
$$

where

$$
\begin{gathered}
(M x)(t)=-\int_{a}^{b} G_{0}(t, s) \sum_{i=1}^{m} p_{i}(s) x\left(s-\tau_{i}(s)\right) d s, \\
\psi(t)=\int_{a}^{b} G_{0}(t, s) f(s) d s .
\end{gathered}
$$

Using Theorem 9, we obtain that the operator $M$ is positive.

The conditions of Theorem 7 imply that $\|M\|<1$.

Now it is clear that

$$
\begin{aligned}
x(t)= & (I-M)^{-1} \psi(t)=\psi(t)+(M \psi)(t) \\
& +\left(M^{2} \psi\right)(t)+\cdots
\end{aligned}
$$

It follows from the positivity of the operator $M$ that for every nonnegative $f$ we get according to $(78) \psi(t) \geq 0$ and $x(t) \geq \psi(t) \geq 0$.

It is clear that

$$
\begin{aligned}
0 & \leq x(t)-\psi(t) \\
& =\int_{a}^{b} G(t, s) f(s) d s-\int_{a}^{b} G_{0}(t, s) f(s) d s \\
& =\int_{a}^{b}\left[G(t, s)-G_{0}(t, s)\right] f(s) d s .
\end{aligned}
$$


From nonnegativity of $x(t)$ for every nonnegative $f(t)$, we obtain that $G(t, s) \geq G_{0}(t, s) \geq 0$.

Theorem 11 has been proven.

Theorem 12. Let conditions (40) and (76) be fulfilled, $p_{i}(t) \geq$ 0 for $i=1, \ldots, m$ then problem (1)-(5) is unique solvable and its Green's function $G(t, s)$ is negative for $t, s \in[a, b]$.

We prove this assertion analogously to the proof of Theorem 11.

The proofs of the following two assertions follow from the construction of Green's functions.

Theorem 13. Let

$$
\begin{array}{cc}
0<\beta_{i}, \quad 1 \leq i \leq k, & \frac{J_{1}(\beta, \gamma)}{J(\beta, \gamma)}>1, \\
\frac{J_{j}(\beta, \gamma)}{J(\beta, \gamma)}>\frac{1}{\prod_{i=1}^{j-1} \beta_{i}} \quad 2 \leq j \leq k+1 ;
\end{array}
$$

then Green's function of problem (29), (7) $W_{0}(t, s)$ is negative for $t, s \in[a, b]$.

\section{Theorem 14. Let}

$$
0<\beta_{i}, \quad 1 \leq i \leq k, \quad \max _{1 \leq i \leq k+1} J(\beta, \gamma) \cdot J_{i}(\beta, \gamma)<0
$$

then Green's function of problem (29), (7) $W_{0}(t, s)$ is positive for $t, s \in[a, b]$.

The proof of the following two theorems can be obtained analogously from the proof of Theorem 11.

Theorem 15. Let conditions (60) and (82) be fulfilled, $p_{i}(t) \leq$ 0 for $i=1, \ldots, m$; then problem (1)-(4), (7) is unique solvable and its Green's function $W(t, s)$ is positive for $t, s \in[a, b]$.

Theorem 16. Let conditions (60) and (81) be fulfilled, $p_{i}(t) \geq 0$ for $i=1, \ldots, m$; then problem (1)-(4), (7) is unique solvable and its Green's function $W(t, s)$ is negative for $t, s \in[a, b]$.

\section{Conflict of Interests}

The authors declare that they have no conflict of interests regarding the publication of this paper.

\section{References}

[1] N. V. Azbelev, V. P. Maksimov, and L. F. Rakhmatullina, Introduction to the Theory of Functional Differential Equations, Advanced Series in Mathematical Science and Engineering, World Federation, Atlanta, Ga, USA, 1995.

[2] D. Baĭnov and P. Simeonov, Impulsive Differential Equations, vol. 66 of Pitman Monographs and Surveys in Pure and Applied Mathematics, Longman Scientific \& Technical, Harlow, UK, 1993.

[3] V. Lakshmikantham, D. D. Baĭnov, and P. S. Simeonov, Theory of Impulsive Differential Equations, vol. 6 of Series in Modern Applied Mathematics, World Scientific, Singapore, 1989.
[4] S. G. Pandit and S. G. Deo, Differential Systems Involving Impulses, vol. 954 of Lecture Notes in Mathematics, Springer, Berlin, Germany, 1982.

[5] A. M. Samoilenko and N. A. Perestyuk, Differential Equations with Impulse Effect, Villa Skola, Kiev, Russia, 1987, (Russian).

[6] A. M. Samoilenko and A. N. Perstyuk, Impulsive Differential Equations, World Scientific, Singapore, 1992.

[7] S. T. Zavalishchin and A. N. Sesekin, Dynamic Impulse Systems: Theory and Applications, vol. 394 of Mathematics and Its Applications, Kluwer Academic Publishers, Dordrecht, The Netherlands, 1997.

[8] M. Federson and S. Schwabik, "Generalized ODE approach to impulsive retarded functional differential equations," Differential and Integral Equations, vol. 19, no. 11, pp. 1201-1234, 2006.

[9] M. Federson and S. Schwabik, "A new approach to impulsive retarded differential equations: stability results," Functional Differential Equations, vol. 16, no. 4, pp. 583-607, 2009.

[10] Z. Halas and M. Tvrdý, "Continuous dependence of solutions of generalized linear differential equations on a parameter," Functional Differential Equations, vol. 16, no. 2, pp. 299-313, 2009.

[11] J. Kurzweil, "Generalized ordinary differential equations and continuous dependence on a parameter," Czechoslovak Mathematical Journal, vol. 7, no. 82, pp. 418-449, 1957.

[12] S. Schwabik, Generalized Ordinary Differential Equations, Eorkd Scientific, Singapore, 1992.

[13] M. Tvrdý, "Generalized differential equations in the space of regulated functions (boundary value problems and controllability)," Mathematica Bohemica, vol. 116, no. 3, pp. 225-244, 1991.

[14] M. Tvrdý, "Differential and integral equations in the space of regulated functions," Memoirs on Differential Equations and Mathematical Physics, vol. 25, pp. 1-104, 2002.

[15] M. Ashordia, "Criteria of correctness of linear boundary value problems for systems of generalized ordinary differential equations," Czechoslovak Mathematical Journal, vol. 46, no. 1213, pp. 385-404, 1996.

[16] A. Domoshnitsky and M. Drakhlin, "On boundary value problems for first order impulse functional-differential equations," in Boundary Value Problems for Functional Differential Equations, J. Henderson, Ed., pp. 107-117, World Scientific, Singapore, 1995.

[17] A. Domoshnitsky and M. Drakhlin, "Nonoscillation of first order impulse differential equations with delay," Journal of Mathematical Analysis and Applications, vol. 206, no. 1, pp. 254269, 1997.

[18] A. Domoshnitsky, M. Drakhlin, and E. Litsyn, "On N-th order functional-differential equations with impulses," in Memoirs on Differential Equations and Mathematical Physics, vol. 12, pp. 5056, 1997.

[19] A. Domoshnitsky, "Boundary value problem for first order impulsive functional-differential nonlinear equations," Functional Differential Equations, vol. 4, pp. 37-45, 1997.

[20] A. Domoshnitsky, M. Drakhlin, and E. Litsyn, "On boundary value problems for $\mathrm{N}$-th order functional differential equations with impulses," Advances in Mathematical Sciences and Applications, vol. 8, no. 2, pp. 987-996, 1998.

[21] D. D. Baĭnov, S. G. Hristova, S. C. Hu, and V. Lakshmikantham, "Periodic boundary value problems for systems of first order impulsive differential equations," Differential and Integral Equations, vol. 2, no. 1, pp. 37-43, 1989. 
[22] J. Li, J. J. Nieto, and J. Shen, "Impulsive periodic boundary value problems of first-order differential equations," Journal of Mathematical Analysis and Applications, vol. 325, no. 1, pp. 226-236, 2007.

[23] J. J. Nieto and R. Rodríguez-López, "Periodic boundary value problem for non-Lipschitzian impulsive functional differential equations," Journal of Mathematical Analysis and Applications, vol. 318, no. 2, pp. 593-610, 2006.

[24] S. C. Hu and V. Lakshmikantham, "Periodic boundary value problems for second order impulsive differential systems," Nonlinear Analysis. Theory, Methods \& Applications, vol. 13, no. 1, pp. 75-85, 1989.

[25] R. P. Agarwal, L. Berezansky, E. Braverman, and A. Domoshnitsky, Nonoscillation Theory of Functional Differential Equations with Applications, Springer, 2012.

[26] V. Lakshmikantham and X. Liu, "On quasistability for impulsive differential systems," Nonlinear Analysis. Theory, Methods \& Applications, vol. 13, no. 7, pp. 819-828, 1989.

[27] M. Benchohra, J. Henderson, and S. K. Ntouyas, Impulsive Differential Equations and Inclusions, vol. 2 of Contemporary Mathematics and Its Applications, Hindawi Publishing Corporation, New York, NY, USA, 2006.

[28] A. L. Skubachevskii, "Nonclassical boundary value problems," Journal of Mathematical Sciences, vol. 155, no. 2, pp. 199-330, 2008.

[29] M. Benchohra, J. Henderson, and S. K. Ntouyas, "An existence result for first-order impulsive functional differential equations in Banach spaces," Computers \& Mathematics with Applications, vol. 42, no. 10-11, pp. 1303-1310, 2001.

[30] Z. Fan, "Impulsive problems for semilinear differential equations with nonlocal conditions," Nonlinear Analysis. Theory, Methods \& Applications, vol. 72, no. 2, pp. 1104-1109, 2010.

[31] Z. Fan and G. Li, "Existence results for semilinear differential equations with nonlocal and impulsive conditions," Journal of Functional Analysis, vol. 258, no. 5, pp. 1709-1727, 2010.

[32] S. Ji and G. Li, "Existence results for impulsive differential inclusions with nonlocal conditions," Computers \& Mathematics with Applications, vol. 62, no. 4, pp. 1908-1915, 2011.

[33] S. Ji and S. Wen, "Nonlocal Cauchy problem for impulsive differential equations in Banach spaces," International Journal of Nonlinear Science, vol. 10, no. 1, pp. 88-95, 2010.

[34] S. Ji and G. Li, "A unified approach to nonlocal impulsive differential equations with the measure of noncompactness," Advances in Difference Equations, vol. 2012, article 182, 2012.

[35] L. Zhu, Q. Dong, and G. Li, "Impulsive differential equations with nonlocal conditions in general Banach spaces," Advances in Difference Equations, vol. 2012, article 10, 2012. 


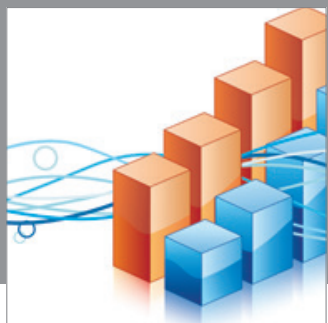

Advances in

Operations Research

mansans

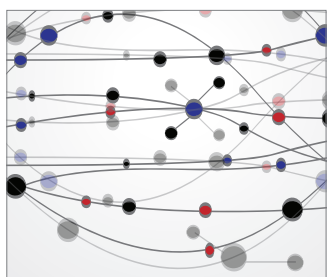

The Scientific World Journal
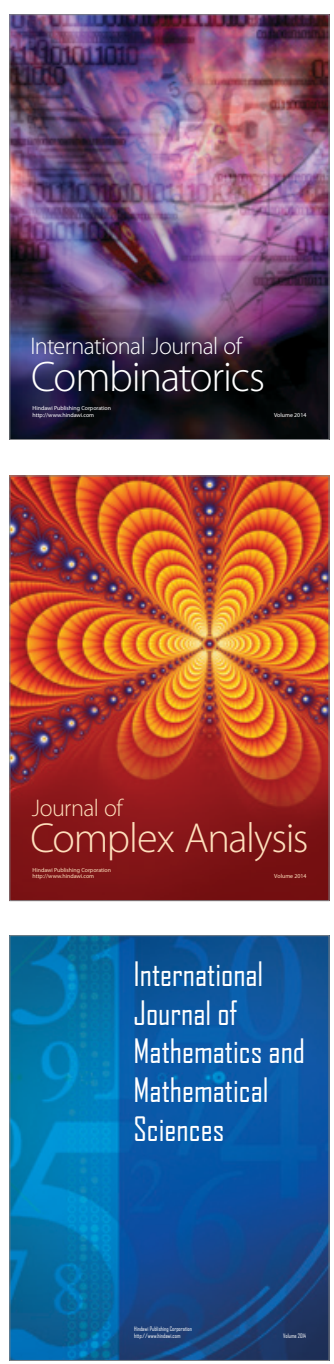
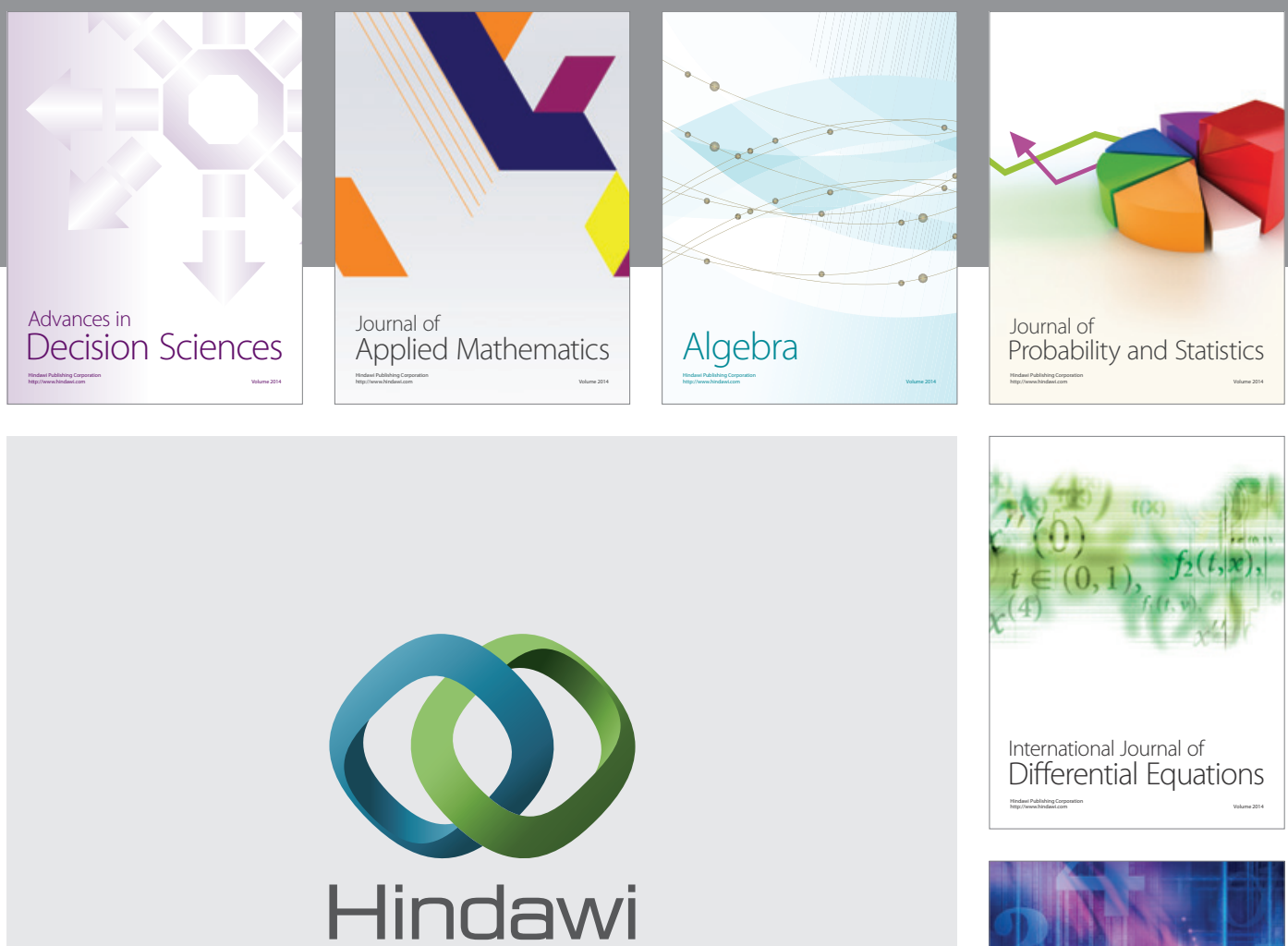

Submit your manuscripts at http://www.hindawi.com
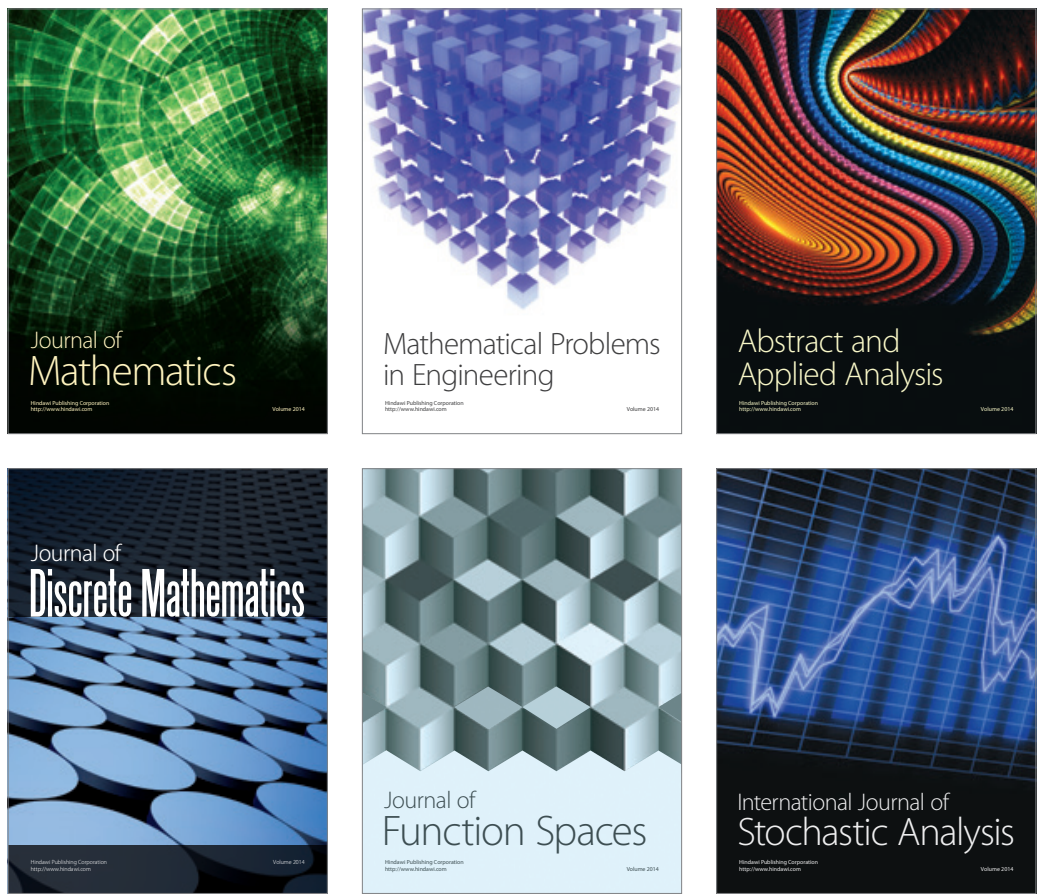

Journal of

Function Spaces

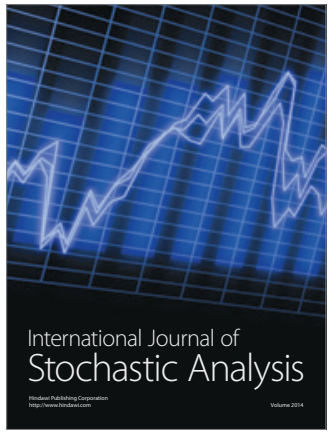

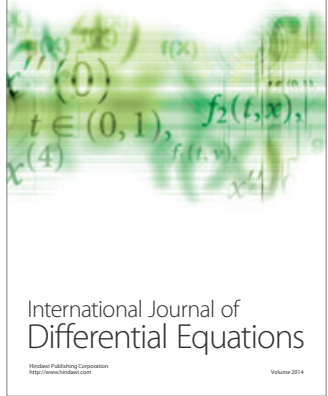
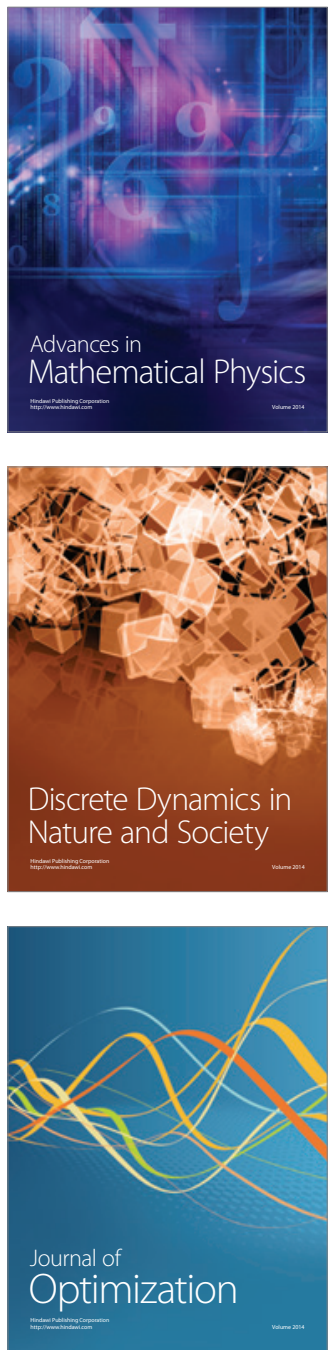